\title{
Small Interfering RNA (siRNA) against the Survivin Gene Increases Apoptosis in a Canine Melanoma Cell Line
}

\author{
Miyuki MORIYAMA $^{1)}$, Rui KANO ${ }^{1) *}$, Haruhiko MARUYAMA ${ }^{1)}$, Atsuhiko HASEGAWA ${ }^{2)}$ and Hiroshi KAMATA ${ }^{1)}$ \\ ${ }^{1)}$ Department of Pathobiology, Nihon University School of Veterinary Medicine, Fujisawa, Kanagawa 252-0880 and ${ }^{2)}$ Teikyo University \\ Institute of Medical Mycology, 539 Otsuka, Hachioji, Tokyo 192-0395, Japan
}

(Received 23 March 2010/Accepted 18 July 2010/Published online in J-STAGE 2 August 2010)

\begin{abstract}
The effect of down-regulation of survivin expression by small interfering RNA (siRNA) against the canine survivin gene on apoptosis was investigated by transfecting MCM-N1 (a canine malignant oral melanoma cell line) cells with siRNA using cationic liposomes. The siRNA against the canine survivin gene induced an increase in the rate of apoptotic cells and a decrease in the number of viable cells. We also measured sequence-specific down-regulation of survivin expression by reverse transcription-PCR and western blot analysis. The siRNA directed against survivin reduced both mRNA and protein expression in MCM-N1 cells. These findings suggest the importance of survivin in canine melanoma tumors for inducing apoptosis, and reinforce the possibility of using survivin as a putative therapeutic target in canine malignant melanoma tumor.
\end{abstract}

KEY WORDS: apoptosis, canine malignant melanoma, small interfering RNA, survivin.

J. Vet. Med. Sci. 72(12): 1643-1646, 2010

Canine malignant melanoma is a spontaneous, highly aggressive neoplasm that can readily metastasize to the other organs [7, 17]. Systemic chemotherapy for canine malignant melanoma has shown poor prognosis and more effective therapies are required. Several novel therapeutic modalities have been reported, including methods to enhance immunosurveillance (e.g., tumor vaccines and a DNA vaccine) [7, 17]. In addition, immunomodulation therapy is currently used in veterinary oncology [17]; however, more investigation in clinical fields is required.

Survivin is a strong anti-apoptotic protein that is expressed in human advanced melanomas [2, 4, 14]. Because of the ability of this protein to decrease the apoptotic response to cytotoxic chemotherapy, survivin in human melanomas has been a target for the development of survivin inhibitors [8]. Some studies have suggested that survivin over-expression might be associated with malignancy of canine tumors $[10,11]$ and thus canine survivin may be an important therapeutic target in canine melanoma, as well as an unfavorable prognostic marker $[10,11,16]$.

In our previous studies, we showed that siRNA directed against the anti-apoptotic genes Bcl-2 and Mcl-1 reduced their mRNA and protein expression in a canine mammary gland tumor cell line, CF33, and in the melanoma cell line MCM-N1, and that the number of viable cells decreased with an increase in the apoptotic cell rate $[6,9,13]$. Those findings indicated that siRNA is a useful tool with which to evaluate therapeutic targets, even in canine tumor cells.

In the present study, we evaluated siRNA targeting canine survivin as a novel approach to down-regulate survivin in canine melanoma cells. The use of specific siRNA led to a moderate increase in apoptotic cell death and inhibi-

\footnotetext{
* Correspondence to: Kano, R., Department of Pathobiology, Nihon University School of Veterinary Medicine, 1866 Kameino, Fujisawa, Kanagawa 252-8510, Japan.

e-mail: kano@brs.nihon-u.ac.jp
}

tion of cell growth.

The canine malignant oral melanoma cell line MCM-N1 (DS Pharma Biomedical Co., Ltd., Osaka, Japan) was maintained in Dulbecco's modified Eagle's medium (DMEM) (Sigma-Aldrich, Tokyo, Japan) supplemented with 10\% FBS at $37^{\circ} \mathrm{C}$ in a humidified atmosphere containing $5 \%$ $\mathrm{CO}_{2}$.

For silencing the canine survivin gene, 5 siRNA sequences were designed from the canine survivin gene (canine survivin gene; Genbank accession No. AY741504) using the Selection Program (Whitehead Institute for Biomedical Research siRNA, http://jura.wi.mit.edu/bioc/siRNAext/). Searches of the genome database (BLAST, http:// blast.ddbj.nig.ac.jp/top-j.html) were carried out to ensure that these sequences would not target any other canine genes. siRNA targeting the specific sequence 5'-CAA GCA GAA AGA ATT CGA A -3' (405-423 bp) was selected to down-regulate survivin expression in this study based on its effectiveness in preliminary studies (data not shown). A non-targeting RNA (ntRNA) sequence, which does not target any known mammalian gene, was designed by Qiagen and used as the negative control. The target sequence of the ntRNA (5'-UUC UCC GAA CGC GUC ACG U-3') had no matches with mammalian genes [1]. The siRNA and ntRNA were synthesized using the Silencer siRNA Construction Kit (Ambion, Tokyo, Japan) according to manufacturer's instructions.

Transfection with siRNA and ntRNA was performed as previously described [9, 15] using cationic liposomes (siLentFect reagent, Bio-Rad Laboratories, Tokyo, Japan) with MCM-N1 cells at approximately $50 \%$ confluency in 96- and 6-well plates, according to the manufacturer's instructions. The mock transfection contained only the reagent (cationic liposomes). The transfected cells were examined based on apoptotic cell rates, viability analysis, reverse transcription-PCR (RT-PCR), and western blotting. 
All experiments were performed in triplicate.

The findings for apoptotic cell rates and viability analyses are presented as mean \pm SEM (standard error of the mean) and were analyzed by one-way analysis of variance (ANOVA) and corrected for multiple comparisons using the Bonferroni method. Statistical significance $(P<0.05)$ was analyzed using the Excel Statistical Program File ystat2002 program (Igaku Tosho Shuppan, Tokyo, Japan).

Apoptotic cells were detected by the terminal deoxynucleotidyl transferase (TdT)-mediated dUTP-biotin nick end labeling (TUNEL) method. The TUNEL method was performed according to the protocol of the DeadEnd ${ }^{\mathrm{TM}}$ Colorimetric Apoptosis Detection System (Promega Corp., Madison, WI, U.S.A.). At least 500 cells were counted using a light microscope. The viability assays for transfected cells were evaluated as described previously $[9,15]$. After the 48-hr transfection, apoptotic cell rates were determined by immunohistochemical analysis of ssDNA. The apoptotic cell rates for the anti-survivin siRNA transfection, ntRNA transfection, mock transfection and medium alone were $34.8 \pm 5.8,3.6 \pm 1.8,2.8 \pm 2.4$ and $2.1 \pm 1.0 \%$, respectively. The increase in apoptosis between cells transfected with siRNA and those transfected with ntRNA was statistically significant $(P<0.05)$.

The viability assays for transfected cells were evaluated as described previously $[9,15]$. After the 48 -hr transfection, the total number of viable cells in the anti-survivin siRNA transfection, ntRNA transfection, mock transfection and for medium alone was $2.09 \pm 0.55 \times 10^{5} / \mathrm{ml}, 3.32 \pm 0.07 \times 10^{5} /$ $\mathrm{ml}, 3.17 \pm 0.22 \times 10^{5} / \mathrm{m} l$ and $3.36 \pm 0.24 \times 10^{5} / \mathrm{ml}$, respectively (Fig. 1). There was a statistically significant difference in viable cell rate between cells transfected with siRNA and those transfected with ntRNA $(P<0.05)$.

After the 48-hr transfection, total RNA was extracted from the cells using the RNeasy Mini Kit (Qiagen, Tokyo, Japan) and then treated to remove contaminating DNA

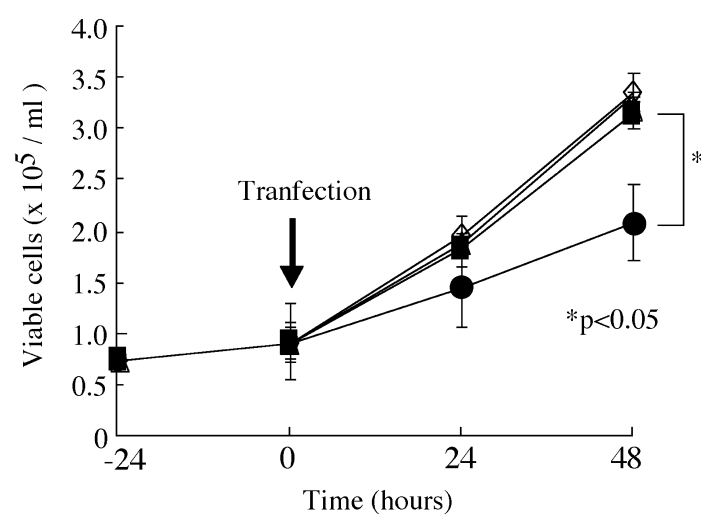

Fig. 1. The total viable cell rates after transfection with siRNA, ntRNA, mock and medium alone. Data represent mean \pm SEM. Results represent the mean \pm SEM. anti-survivin siRNA (siRNA) transfection; $\boldsymbol{\Delta}$, non-targeting RNA (ntRNA) transfection; $>$, mock transfection containing only reagent (cationic liposomes); and $\diamond$, culture in medium alone (Medium). using a DNA-free kit (Ambion). Reverse transcription of the poly $(\mathrm{A})^{+}$RNAs (using $0.5 \mu \mathrm{g}$ ) was performed using Oligo dT primer and the Omniscript Reverse Transcriptase kit (Qiagen).

Reverse transcription-PCR (RT-PCR) for survivin and GAPDH (glyceraldehydes-3-phosphate dehydrogenase) was performed using specific pairs of primers. The primer sequences and gene accession numbers are as follows: Survivin (forward: 5'-ACC ACC GCA TCT CTA CAT TC and reverse: 5'-ACA GAA AGG AAA GCA CAA CC, AY741504) and GAPDH (forward: 5'-GGA GAA AGC TGC CAA ATA TG and reverse: 5'-ACC AGG AAA TGA GCT TGA CA, AB038240). GAPDH was used as an internal control for PCR amplification [5].

The cDNA samples were amplified by PCR in $32 \mu l$ of reaction mixture containing a pair of primers $(0.5 \mu \mathrm{M}$ each), 1.5 units of Taq polymerase (Takara, Kyoto, Japan) and the reagents recommended by the manufacturer. PCR amplification consisted of 27 cycles of template denaturation $\left(94^{\circ} \mathrm{C}, 30 \mathrm{sec}\right)$, primer annealing $\left(60^{\circ} \mathrm{C}, 30 \mathrm{sec}\right)$ and polymerization $\left(72^{\circ} \mathrm{C}, 30 \mathrm{sec}\right)$. Then, $10 \mu l$ of PCR products was separated in a $2 \%(\mathrm{wt} / \mathrm{vol})$ agarose gel, stained with ethidium bromide, and visualized with UV light.

After amplification by RT-PCR, survivin mRNA was detected in MCM-N1 cells (Fig. 2). The level of survivin transcript in MCM-N1 cells decreased when cultured with the siRNA for the canine survivin gene rather than ntRNA (Fig. 2), confirming siRNA inhibition of survivin mRNA expression in this malignant melanoma cell line.

After the 48-hr transfection, the cells were detached by trypsin treatment and proteins were extracted and transferred onto membranes as described previously $[9,15]$. Membranes were blocked for $1 \mathrm{hr}$ with $2 \%$ nonfat milk in PBST (PBS, Wako, Osaka, Japan; and 0.1\% Tween 20) and

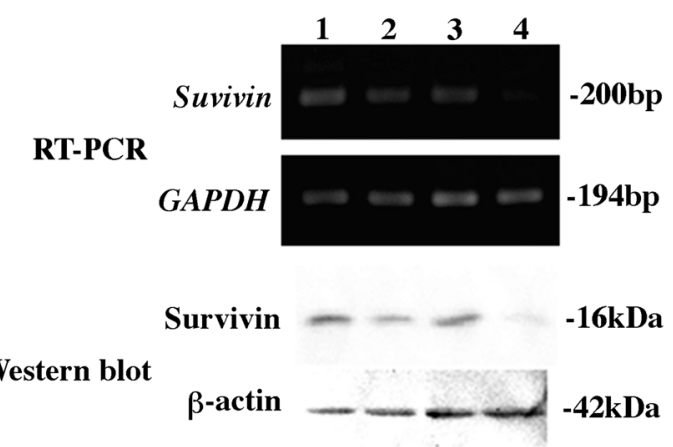

Fig. 2. Reverse transcription-PCR (RT-PCR) analysis of survivin with GAPDH as the internal control. A lower survivin mRNA level was found in cells transfected with anti-survivin siRNA. Western blot analysis of survivin protein compared with beta-actin as the internal control. A significantly lower level of survivin protein was detected in cells transfected with siRNA and detected by anti-survivin antibody. Lane 1, culture in medium; lane 2 , mock transfection containing only reagent (cationic liposomes); lane 3, non-targeting RNA transfection; and lane 4, anti-survivin siRNA transfection. 
then cut into two pieces. One piece was incubated with antisurvivin monoclonal antibody (1:200 dilution, sc17779 Santa Cruz Biotechnology, Santa Cruz, CA, U.S.A.) overnight at $4^{\circ} \mathrm{C}$. After washing with PBST, the membrane was incubated with secondary antibody (1:5,000 dilution, 238569 , Promega). The other membrane piece was incubated with anti- $\beta$-actin monoclonal antibody $(1: 5,000$, Sigma-Aldrich) [3], washed with PBST, and then incubated with secondary antibody (1:5,000 dilution, 238569, Promega) under the same conditions as for the anti-survivin antibody. $\beta$-Actin was used as the internal control to ensure equal protein extraction from cells. After washing with PBST, immunoreactive proteins were detected by ECL (ECK Plus Western Blotting Detection System, GE Healthcare UK Ltd., Little Chalfont, U.K.).

Expression of survivin protein, as determined by western blot analysis, was found to be significantly lower in cells transfected with siRNA, whereas there were no changes in cells transfected with ntRNA, both in the mock reactions and with medium alone (Fig. 2). Therefore, this siRNA inhibited expression of survivin protein in MCM-N1 cells.

Our findings demonstrate that siRNA directed against the canine survivin gene reduces both its $\mathrm{mRNA}$ and protein expression in the canine melanoma cell line MCM-N1 (Fig. 2). Furthermore, this siRNA against the canine survivin gene decreased the number of viable cells and increased the apoptotic cell rate (Fig. 1). We also detected survivin expression in canine mammary tumors and melanoma tissue by immunohistochemical analysis (data not shown). In one of our previous studies, autoantibodies to survivin were detected in $27.1 \%$ of cancer dogs, and this represented a significant difference from control populations consisting of normal and non-cancer disease dogs [13]. The highest positivity rates $(>50 \%)$ among cancer cases were seen in dogs with mammary tumor, squamous cell carcinoma and melanoma, suggesting that canine survivin is expressed in many canine melanoma tissues [13]. The present study provides further evidence of the importance of survivin in canine melanoma for inducing apoptosis, and reinforces the notion of survivin as a putative therapeutic target in tumors. Ryan et al. [12] proposed that survivin is a more attractive target than other anti-apoptotic proteins for anti-cancer therapy for the following reasons: 1 , the low survivin expression in most normal cells and upregulation in malignant tissue; 2, survivin is a nodal protein, i.e., involved in multiple signaling mechanisms controlling tumor maintenance; 3 , targeting survivin may block angiogenesis as well as tumor cell growth; and 4, survivin is responsible for resistance to multiple types of anticancer therapy [12]. We also believe that inhibition of survivin may aid the prevention of canine tumors.

Several novel experimental therapeutic strategies have been developed to target human survivin. These include anti-sense oligonucleotides, ribozymes, and siRNA molecules targeting survivin, as well as small molecule inhibitors of survivin function [8]. One such candidate is YM155 (Astellas Pharma), a small molecule that selectively inhibits survivin mRNA transcription and protein expression in several human tumor cell lines, including melanoma [8]. YM155 at the recommended dose, as well as its provocative antitumor efficacy, prompted a phase II evaluation of this compound in melanomas [8].

Thus, additional experiments are warranted to investigate small molecule inhibitors of survivin function in canine melanoma.

ACKNOWLEDGMENTS. This study was supported by grants from the Academic Frontier Project of the Ministry of Education, Culture, Sports, Science and Technology (MEXT) of Japan, Nihon University and Kariya Animal Hospital.

\section{REFERENCES}

1. Beardsley, A., Fang, K., Mertz, H., Castranova, V., Friend, S. and Liu, J. 2005. Loss of caveolin-1 polarity impedes endothelial cell polarization and directional movement. J. Biol. Chem. 280: $3541-3547$.

2. Calder, K. B., Khalil, F. K., Schauder, S., Cualing, H. D. and Morgan, M. B. 2008. Immunohistochemical expression of survivin in cutaneous sebaceous lesions. Am. J. Dermatopathol. 30: $545-548$.

3. Danilkovitch-Miagkova, A., Miagkov, A., Skeel, A., Nakaigawa, N., Zbar, B. and Leonard, E. J. 2001. Oncogenic mutants of RON and MET receptor tyrosine kinases cause activation of the catenin pathway. Mol. Cell. Biol. 21: 5857-5868.

4. Ding, Y., Prieto, V. G., Zhang, P. S., Rosenthal, S., Smoth, K. K., Skelton, H. G. and Diwan, A. H. 2006. Nuclear expression of the antiapoptic protein survinin in malignant melanoma. Cancer 106: 1123-1129.

5. Grone, A., Weckmann, M. T., Capen, C. C. and Rosol, T. J. 1996. Canine glyceraldehydes-3-phosphate dehydrogenase complementary DNA: polymerase chain reaction amplification, cloning, partial sequence analysis, and use as loading control in ribonuclease protection assays. Am. J. Vet. Res. 57: 254 257.

6. Kano, R., Yano, T., Nagamatsu, K., Maruyama, H., Kamata, H. and Hasegawa, A. 2009. Effectiveness of small interfering RNA (siRNA) against the $M c l-1$ gene in a canine mammary gland tumor cell line. Res. Vet. Sci. 87: 64-66.

7. Liao, J. C. F., Gregor, P., Wolchok, J. D., Orlandi, F., Craft, D., Leung, C., Houghton, A. N. and Bergman, P. J. 2006. Vaccination with human tyrosinase DNA induces antibody response in dogs with advanced melanoma. Cancer Immun. 6: 8 .

8. Mita, A. C., Mita, M. M., Nawrocki, S. T. and Giles, F. J. 2008. Survivin: key regulator of mitosis and apoptosis and novel target for cancer therapeutics. Clin. Cancer Res. 14: 5000-5005.

9. Nagamatsu, K., Tsuchiya, F., Oguma, K., Maruyama, H., Kano, R. and Hasegawa, A. 2006. The effect of small interfering RNA (siRNA) against the Survivin gene on apoptosis and chemosensitivity in a canine mammary gland tumore cell line. Res. Vet. Sci. 84: 49-55.

10. Rankin, W. V., Henry, C. J., Turnquist, S. E., Turk, J. R., Beissenherz, M. E., Tyler, J. W. and Green, J. A. 2008. Comparison of distribution of surviving among tissues from urinary bladders of dogs with cystitis, transitional cell carcinoma, or histologically normal urinary bladders. Am. J. Vet. Res. 69: 10731078 . 
11. Rebhun, R. B., Lana, S. E., Ehrhart, E. K., Charles, J. B. and Thamm, D. H. 2008. Comparative analysis of surviving expression in united and relapsed canine lymphoma. J. Vet. Int. Med. 22: 989-995.

12. Ryan, B. M., O’Donovan, N. and Duffy, M. J. 2009. Survivin: a new target for anti-cancer therapy. Cancer Treat. Rev. 25: $553-562$.

13. Tango, Y., Kano, R., Maruyama, H., Asano, K., Tanaka, S., Hasegawa, A. and Kamata, H. 2010. Detection of autoantibodies against survivin in sera from cancer Dogs. J. Vet. Med. Sci. (in press).

14. Vetter, C. S., Muller-Blech, K., Schrama, D., Brocker, E. and Becker, J. C. 2005. cytoplasmic and nuclear expression of survivin in melanocytic skin lesions. Arch. Dermatol. Res. 297:
26-30.

15. Watanabe, Y., Kano, R., Maruyama, H., Hasegawa, A. and Kamata, H. 2010. Small interfering RNA (siRNA) against the Bcl-2 gene increases apoptosis in a canine melanoma cell line. J. Vet. Med. Sci. 72: 383-386.

16. Wimmershoff, J., Polkinghorne, A., Grest, P., Schade, B., Marchal, T., Keller, S. M. and Guscetti, F. 2010. Immunohistochemical detection of survivin in canine lymphoma. J. Comp. Pathol. (in press) (doi:10.1016/j.jcpa.2009.12.004.)

17. Withrow, S. J. and Vail, D. M. 2007. Melanocytic tumors (melanocytic nervus, melanocytoma, melanosarcoma, malignant melanoma) pp. 389-393. In: Withrow \& MacEwen's Small Animal Clinical Oncology, 4th ed., Saunders Elsevier, St. Louis. 